\title{
Apparent Diffusion Coefficient and Transient Neurological Deficit after Revascularization Surgery in Moyamoya Disease.
}

\section{AUTHOR(S):}

Araki, Yoshio; Takagi, Yasushi; Fushimi, Yasutaka; Arakawa, Yoshiki; Funaki, Takeshi; Kikuchi, Takayuki; Takahashi, Jun C; Togashi, Kaori; Miyamoto, Susumu

\section{CITATION:}

Araki, Yoshio ...[et al]. Apparent Diffusion Coefficient and Transient Neurological Deficit after Revascularization Surgery in Moyamoya Disease.. Journal of stroke and cerebrovascular diseases 2015, 24(9): 2054-2059

\section{ISSUE DATE:}

2015-09

\section{URL:}

http://hdl.handle.net/2433/202246

\section{RIGHT:}

(c) 2015. This manuscript version is made available under the CC-BY-NC-ND 4 . 0 license

http://creativecommons.org/licenses/by-nc-nd/4.0/; The full-text file will be made open to the public on 10 July 2016 in accordance with publisher's 'Terms and Conditions for Self-Archiving'.; This is not the published version. Please cite only the published version; この論文は出版社版でありません。引用の際には出版社版をご確認ご利用ください。 
Apparent diffusion coefficient and transient neurological deficit after revascularization surgery in moyamoya disease

Yoshio Araki, MD, $\mathrm{PhD}^{1}$, Yasushi Takagi, $\mathrm{MD}, \mathrm{PhD}^{1}$, Yasutaka Fushimi, $\mathrm{MD}, \mathrm{PhD}^{2}$, Yoshiki Arakawa, MD, $\mathrm{PhD}^{1}$, Takeshi Funaki, $\mathrm{MD}, \mathrm{PhD}^{1}$, Takayuki Kikuchi, $\mathrm{MD}, \mathrm{PhD}^{1}$, Jun C Takahashi, MD, $\mathrm{PhD}^{1}$, Kaori Togashi, $\mathrm{MD}, \mathrm{PhD}^{2}$, Susumu Miyamoto, $\mathrm{MD}, \mathrm{PhD}^{1}$

1. Department of Neurosurgery, Kyoto University Graduate School of Medicine

2. Department of Diagnostic Imaging and Nuclear Medicine, Kyoto University Graduate School of Medicine

\section{Corresponding author:}

Yoshio Araki, MD, PhD

Department of Neurosurgery, Kyoto University Graduate School of Medicine, 54

Kawahara-cho, Shogoin, Sakyo-ku, Kyoto 606-8507, Japan

E-mail: yaraki@kuhp.kyoto-u.ac.jp 
Phone: +81-75-751-3459

Fax: +81-75-752-9501

Running title: ADC and transient neurological deficit in MMD 


\section{ABSTRACT}

Background: Transient neurological deficits are relatively common after direct revascularization surgery for moyamoya disease. Although recent evidence has revealed the clinical features and pathophysiology, preoperative predictors have remained unclear. This study investigated whether the apparent diffusion coefficient in normal-appearing white matter on magnetic resonance imaging could offer a predictor of postoperative transient neurological deficits. Methods: This study included adult patients with moyamoya disease who underwent superficial temporal artery-middle cerebral artery bypass. Preoperative apparent diffusion coefficients were measured in normal-appearing white matter on ipsilateral hemisphere. Single photon emission computed tomography with ${ }^{123}$ I-iodoamphetamine was performed on postoperative day 2-6. Relationships between mean apparent diffusion coefficient and postoperative transient neurological deficits were assessed. Results: Twenty of the 26 subjects (76.9\%) experienced transient neurological deficits. Focal hyperemia on postoperative single photon emission computed tomography appeared in 11 of the 20 subjects with postoperative transient neurological deficits and 1 of the 6 subjects without $(P=0.12)$. Mean apparent 
diffusion coefficients in patients with and without postoperative transient neurological deficits were $748.3 \pm 12 \mathrm{~mm}^{2} / \mathrm{s} \times 10^{-6}$ and $679.7 \pm 21.9 \mathrm{~mm}^{2} / \mathrm{s} \times 10^{-6}$, respectively $(P=.0091)$. Conclusions: Preoperative apparent diffusion coefficient elevation in normal-appearing white matter may indicate postoperative transient neurological deficits not only in patients with postoperative hyperemia on single photon emission computed tomography, but also in patients with this pathology. 


\section{INTRODUCTION}

Moyamoya disease (MMD) is a rare cerebrovascular disease characterized by progressive stenosis or occlusion of bilateral internal carotid arteries in association with development of basal collateral vascular networks.(1) Direct revascularization surgery, such as superficial temporal artery (STA)-middle cerebral artery (MCA) bypass, has been established as a beneficial intervention to increase cerebral blood flow (CBF), guard against ischemic stroke and provide long-term favorable outcomes for ischemic-type MMD.(2) Until recently, whether direct revascularization for patients with hemorrhagic onset could prevent rebleeding has been controversial. Results of the Japan Adult Moyamoya trial revealed that extracranial-intracranial bypass surgery had the potential to prevent rebleeding events in hemorrhagic-type MMD.(3) Direct revascularization can thus play a protective role in the management of this disease. However, this surgery has some potential perioperative complications. Transient neurological deficits have been recognized as a major event developing after surgery.(4,5) Postoperative CBF studies have shown a variety of cerebral hemodynamics, including focal hyperperfusion,(2) no change/normalization and 
hypoperfusion.(6) Such evidence indicates that transient neurological deficits reflect varying hemodynamic statuses after this surgery, so this specific condition needs to be treated based on an analysis of postoperative CBF. However, the lack of efficient preoperative predictors of postoperative symptoms remains problematic. To resolve this issue, parameters from preoperative imaging able to predict these postoperative events are required.

The apparent diffusion coefficient (ADC) from diffusion-weighted imaging (DWI) has been used as a marker of subtle structural changes in normal-appearing white matter (NAWM) on T2-weighted and fluid-attenuated inversion recuperation (FLAIR) imaging.(7,8) Elevated ADC in NAWM has been identified in cases of extracranial internal carotid artery stenosis(8) and ischemic leukoaraiosis,(7) and in the elderly.(9) Recent evidence from a CBF study in patients with MMD has shown that ADC elevation in NAWM correlates with severe reductions in cerebrovascular reserve (CVR).(10-12)

Calviere et al.(10) and Nakamizo et al.(13) indicated a correlation between ADC elevation in NAWM and long-term neurological impairment in patients with MMD, but 
relationships between preoperative $\mathrm{ADC}$ and perioperative transient neurological deficits have not been clarified.

Here, we hypothesized that more severely impaired CVR would be associated with increased susceptibility to postoperative events. This study therefore investigated whether preoperative elevation of ADC in NAWM could provide a predictor of postoperative transient neurological deterioration.

\section{MATERIALS AND METHODS}

\section{Patients}

Data were obtained from charts and radiological records for patients treated at Kyoto University Hospital between August 2009 and February 2012. Twenty-six Japanese patients with angiographically confirmed adult MMD (7 men, 19 women; mean age, 38.3 years; range, 20-52 years) participated in this study. No patients had coexisting disease. All patients were treated with single antiplatelet before revascularization surgery. 


\section{Surgery and postoperative management}

Direct revascularization comprised STA-MCA single bypass in all cases. Indirect revascularization methods were not included. In our institution, the STA graft is usually anastomosed to the superior trunk of the MCA. After the surgery, blood pressure was kept in control with preoperative level. The antiplatelets were restarted postoperative day two or three after no hemorrhagic complication was confirmed on radiological examination.

\section{Measurement of preoperative mean ADC in NAWM}

MRI was conducted using a 3-T scanner (Magnetom Trio; Siemens, Erlangen, Germany) with a 32-channel phased-array head coil. DWI was obtained using a single-shot echo-planar sequence with the following sequence protocol: repetition time, 4200 ms; echo time, $78 \mathrm{~ms}$; field of view, $220 \times 220 \mathrm{~mm}$; matrix, $128 \times 128$; slice thickness, $3 \mathrm{~mm}$; slice gap, $0.99 \mathrm{~mm}$; number of excitations, 3; b=0, $1000 \mathrm{~mm}^{2} / \mathrm{s}$ with 3 principal axes. The resulting DWI and ADC data were transferred to a separate workstation for analysis. ADC data were normalized into Montreal Neurological 
Institute space with the corresponding b0 images and segmented by cortex and white matter using SPM8 software (www.fil.ion.ucl.ac.uk/spm/). Two or three regions of interest (ROIs) were placed on the ADC map in NAWM at the level of centrum semiovale or corona radiata, where a FLAIR axial slice about $50 \mathrm{~mm}$ above the orbitomeatal baseline showed no abnormality on the operated side (Fig. 1). The shape of each ROI was a circle with a diameter of $1 \mathrm{~cm}$. When setting ROIs, areas containing infarction, hemorrhage or apparent artifacts were not selected.

\section{Postoperative evaluation}

Definitions of postoperative transient neurological deficits were: 1) neurological signs, including neurological deficits and/or severe headache; 2) natural relief of deficits in the short term; 3) absence of new stroke or surgery-related complications as confirmed by postoperative MRI. MRI, including MR angiography (MRA), DWI, FLAIR and T2*-weighted imaging, and resting-state ${ }^{123}$ I-iodoamphetamine (IMP)-single photon emission computed tomography (SPECT) were performed on postoperative day 2-6 for all participants. SPECT was acquired at resting state using a 2-head rotating gamma 
camera (Infinia; GE Medical Systems, Milwaukee, USA) with an extended low-energy general-purpose collimator. Patients were asked to keep the eyes closed during scanning. A bolus of N-isopropyl-p- ${ }^{123}$ I-IMP (167 MBq) was administered at the beginning of image acquisition. Data were acquired in a $64 \times 64$ matrix through a $120^{\circ}$ rotation at angle intervals of $4^{\circ}$. Total imaging time was $30 \mathrm{~min}$. Spatial resolution at the center of view was $9.9 \mathrm{~mm}$ in full-width at half-maximum (FWHM) activity. Transverse reconstruction with Ramp and Butterworth filters (cutoff of 0.5 cycles/pixel and order $10)$, and attenuation correction using the method of Chang $(0.07 / \mathrm{cm})$ were applied. FWHM of the collimator was $10 \mathrm{~mm}$. In evaluating the results of SPECT, marked CBF increases in focal vascular territories were defined as focal hyperemia.

\section{Statistical analysis}

Univariate analysis was performed to investigate relationships between mean ADC in NAWM for patients with and without postoperative transient neurological deficits. The Mann-Whitney $U$ test was used to calculate $P$-values for non-parametric data. To determine whether preoperative ADC in NAWM could predict postoperative transient 
neurological deficits, a receiver operating characteristics (ROC) curve was constructed.

Cut-off values were set using the Youden index. Patients were divided into two groups using this cut-off value. Fisher's exact test examined the relationship between focal hyperemia on postoperative SPECT, mean ADC and transient neurological deficits after revascularization. Statistical data were generated using JMP version 10.0.2 software (SAS Institute, Cary, NC). Values of $P<0.05$ were considered statistically significant.

\section{RESULTS}

Among the 26 subjects, 20 (76.9\%) showed transient neurological deficits after revascularization and the remaining 6 patients (23.1\%) did not. Symptoms appeared in the early postoperative period (mean, 3 days; range, 0-7 days) and resolved in the short term. All symptoms appeared contralateral to the side of surgery and are summarized in Table 1. Postoperative MRI, including MRA, DWI and T2*-weighted imaging, revealed all bypasses were patent and no evidence of perioperative stroke events in any patients. Focal hyperemia on postoperative SPECT appeared in 11 of 20 subjects with postoperative transient neurological deficits and in 1 of 6 subjects without $(P=0.12)$. 
Mean ADCs in patients with and without postoperative transient neurological deficits were $748.3 \pm 12 \mathrm{~mm}^{2} / \mathrm{s} \times 10^{-6}$ and $679.7 \pm 21.9 \mathrm{~mm}^{2} / \mathrm{s} \times 10^{-6}$, respectively $(P=0.0091)$. The box-and-whisker plot of this result is shown in Figure 2. Using the Youden index from the ROC curve (705.5; 70\% sensitivity and 83.3\% specificity), a significant relationship between mean ADC and transient neurological deficits after revascularization was identified $(P=0.032$, Table 2).

\section{DISCUSSION}

In this study, preoperatively elevated diffusion in NAWM offered a predictor of postoperative transient neurological deficits in patients with adult MMD. This is the first report to present a relationship between preoperative ADC and transient neurological deficits after revascularization surgery.

ADC elevation has been reported to reflect water diffusivity due to axonal loss with subtle structural changes in NAWM.(7) Such structural damage has been observed in experimental models of chronic hypoperfusion in which white matter shows glial activation, axonal damage and rarefaction with demyelination.(14,15) Conklin et al. 
offered the first evidence that subtle ischemic changes might be present in regions of steal phenomenon in the NAWM of patients with MMD, leading to increased ADC in these areas.(11)

Postoperative transient neurological deterioration is known to be a relatively common event after revascularization surgery. CBF studies have demonstrated that these symptoms result from hemodynamic conversion resulting from surgery. Postoperative hyperperfusion was recognized as the main contributor after STA-MCA bypass and carotid endarterectomy (CEA) for atherosclerotic condition.(16,17) In MMD, postoperative hyperperfusion has recently come to be considered as a cause of transient neurological deterioration.(2) However, Hayashi et al. reported from the results of CBF analysis in pediatric moyamoya cases that perioperative neurological deterioration might be attributable to focal ischemia after successful bypass surgery, called “watershed shift”.(18) Furthermore, in patients with postoperative transient neurological deterioration, postoperative CBF studies have shown either no change or normalization compared with preoperative (9 of 20 subjects (45\%) in this study). Although these differences may originate from "static" and "fluctuating" cerebral hemodynamics in 
atherosclerotic disease and MMD, respectively, the underlying mechanisms remain unclear. This study focused on not only hyperperfusion syndrome, but also relationships with transient neurological deterioration and preoperative ADC. As mentioned above, understanding predictive information that suggests whether postoperative neurological deterioration may occur has clinical significance for proper treatment to avoid permanent neurological sequelae.

On the other hand, postoperative management has a critical role in this disease. Management with the basis of prophylactic blood pressure lowering may resolve transient neurological deficits from hyperemic state after revasculization surgery.(19) But compulsory blood pressure lowering has possibility to induce low cerebral perfusion pressure on untreated region especially in patients with advanced stage. Considering this concern, we have carried "habitual blood pressure" control in the perioperative period.

Previous investigators have mentioned several preoperative factors to predict postoperative transient neurological deterioration. Most such factors correlated with hyperperfusion. Ohue et al. noted that ischemic-onset patients could be suffering 
symptomatic hyperperfusion.(20) On the other hand, Fujiumura et al. suggested that adult-onset and/or hemorrhagic-onset patients carried a higher risk of symptomatic hyperperfusion.(21) The clinical results from estimating postoperative condition have been inconsistent. Uchino et al. reported that a preoperative increase in cerebral blood volume (CBV) as measured on positron emission tomography (PET) might be useful for predicting postoperative radiological and symptomatic hyperperfusion.(22) They suggested that increased CBV could indicate autoregulatory vasodilatation in response to cerebral perfusion pressure reduction and predict an increased oxygen ejection fraction (OEF) in patients with adult MMD, on the basis of previous studies in patients with carotid occlusive disease.(23-25) Several parameters have been suggested to predict postoperative hyperperfusion, which includes as yet unexplained postoperative transient neurological deterioration associated with normalized or unchanged CBF on postoperative SPECT compared to preoperatively.

In the present study, postoperative transient neurological deterioration was accurately predicted using Youden index. More importantly, this model could identify symptoms that were not associated with hyperemia on postoperative SPECT. What these 
symptoms are influenced by is not fully understood, but our data support the hypothesis that preoperatively elevated mean ADC in NAWM could offer a predictor of transient neurological deterioration developing from slight hemodynamic changes that are undetectable on postoperative CBF studies. Furthermore, this is the first report to predict postoperative transient neurological deterioration using preoperative MRI data. Such results are clinically relevant because MRI is available in many facilities, improving applicability in cases where PET or other modality might be unavailable.

Several limitations must be considered when interpreting the results of this study. First, the number of patients was relatively small. The incidence of postoperative neurological deterioration was higher than that in previous reports,(2) and the criteria for patient selection were not objective, so biases might have influenced the results. Ideally, a multicenter, randomized, prospective study should be performed. Second, in this study, data from cases with revascularization surgery on one side were combined with data from those with revascularization surgery on both sides in the analysis; analyzing these data separately would allow a more precise assessment. Improved CBF or CVR following contralateral surgery may impact the mean ADC on the ipsilateral side. This 
may be a critical issue; to evaluate postoperative hemodynamic change that is undetectable using SPECT by ADC comparing with preoperative one and development of transient neurological deficits is essential in the further investigation. Also, it is certainly important implication for clinicians to predict not transient but permanent complication of the surgery. We think final object of our study is to predict permanent complication during perioperative period using preoperative information.

\section{CONCLUSIONS}

This is the first study to predict transient postoperative neurological deterioration using preoperative mean ADC in adult patients with MMD. Elevated ADC may suggest a risk of postoperative symptoms was not correlated with hyperemia on SPECT. To acquire more solid evidence and elucidate the detailed mechanisms underlying postoperative events, further investigation is essential. 


\section{REFERENCES}

1. Suzuki J, Kodama N. Moyamoya disease-a review. Stroke 1983;14:104-109.

2. Fujimura M, Tominaga T. Lessons learned from moyamoya disease: Outcome of direct/indirect revascularization surgery for 150 affected hemispheres. Neurol Med Chir (Tokyo) 2012;52:327-332.

3. Miyamoto S, Yoshimoto $\mathrm{T}$, Hashimoto $\mathrm{N}$, et al. Effects of extracranial-intracranial bypass for patients with hemorrhagic moyamoya disease: Results of the Japan Adult Moyamoya Trial. Stroke 2014;45:1415-1421.

4. Fujimura M, Shimizu H, Inoue T, et al. Significance of Focal Cerebral Hyperperfusion as a cause of transient neurologic deterioration after extracranial-intracranial bypass for moyamoya disease: comparative study with non-moyamoya patients using N-Isopropyl-p-[ $\left.{ }^{123} \mathrm{I}\right]$ Iodoamphetamine single-photon emission computed tomography. Neurosurgery 2011;68:957-964.

5. Hayashi K, Horie N, Suyama K, et al. Incidence and clinical features of symptomatic cerebral hyperperfusion syndrome after vascular reconstruction. World Neurosurg 2012;78:447-454. 
6. Pandey P, Steinberg GK. Neurosurgical advances in the treatment of moyamoya disease. Stroke 2011;42:3304-3310.

7. O'Sullivan M, Summers PE, Jones DK, et al. Normal-appearing white matter in ischemic leukoaraiosis: A diffusion tensor MRI study. Neurology. 2001;57:2307-2310.

8. Soinne L, Helenius J, Saimanen E, et al. Brain diffusion changes in carotid occlusive disease treated with endarterectomy. Neurology 2003;61:1061-1065.

9. Engelter ST, Provenzale JM, Petrella JR, et al. The effect of aging on the apparent diffusion coefficient of normal-appearing white matter. AJR Am J of Roentgenol 2000;175:425-430.

10. Calviere L, Ssi Yan Kai G, Catalaa I, et al. Executive dysfunction in adults with moyamoya disease is associated with increased diffusion in frontal white matter. $\mathrm{J}$ Neurol Neurosurg Psychiatry 2012;83:591-593.

11. Conklin J, Fierstra J, Crawley AP, et al. Impaired cerebrovascular reactivity with steal phenomenon is associated with increased diffusion in white matter of patients with moyamoya disease. Stroke 2010;41:1610-1616. 
12. Jeong H, Kim J, Choi HS, et al. Changes in integrity of normal-appearing white matter in patients with moyamoya disease: A diffusion tensor imaging study. AJNR Am J Neuroradiol 2011;32:1893-1898.

13. Nakamizo A, Kikkawa Y, Hiwatashi A, et al. Executive function and diffusion in frontal white matter of adults with moyamoya disease. J Stroke Cerebrovasc Dis 2013;23:457-461.

14. Kurumatani T, Kudo T, Ikura Y, et al. White matter changes in the gerbil brain under chronic cerebral hypoperfusion. Stroke 1998;29:1058-1062.

15. Shibata M, Ohtani R, Ihara M, et al. White matter lesions and glial activation in a novel mouse model of chronic cerebral hypoperfusion. Stroke 2004;35:2598-2603.

16. Hayashi K, Horie N, Suyama K, et al. Incidence and clinical features of symptomatic cerebral hyperperfusion syndrome after vascular reconstruction. World Neurosurg 2012;78:447-454.

17. Kuroda S, Kamiyama H, Abe H, et al. Temporary neurological deterioration caused by hyperperfusion after extracranial-intracranial bypass-case report and study of cerebral hemodynamics. Neurol Med Chir (Tokyo) 1994;34:15-19. 
18. Hayashi T, Shirane R, Fujimura $\mathrm{M}$, et al. Postoperative neurological deterioration in pediatric moyamoya disease: Watershed shift and hyperperfusion. J Neurosurg Pediatr 2010;6:73-81.

19. Fujimura M, Inoue T, Simizu H, et al. Efficacy of prophylactic blood pressure lowering according to a standardized postoperative management protocol to prevent symptomatic cerebral hyperperfusion after direct revascularization surgery for moyamoya disease. Cerebrovasc Dis 2012;33:436-445.

20. Ohue S, Kumon Y, Kohno K, et al. Postoperative temporary neurological deficits in adults with moyamoya disease. Surg Neurol 2008;69:281-286.

21. Fujimura M, Mugikura S, Kaneta T, et al. Incidence and risk factors for symptomatic cerebral hyperperfusion after superficial temporal artery-middle cerebral artery anastomosis in patients with moyamoya disease. Surg Neurol 2009;71:442-447.

22. Uchino H, Kuroda S, Hirata $\mathrm{K}$, et al. Predictors and clinical features of postoperative hyperperfusion after surgical revascularization for moyamoya disease: A serial single photon emission CT/positron emission tomography study. Stroke 2012;43:2610-2616. 
23. Derdeyn CP, Videen TO, Yundt KD, et al. Variability of cerebral blood volume and oxygen extraction: Stages of cerebral haemodynamic impairment revisited. Brain 2002;125:595-607.

24. Fukuda T, Ogasawara K, Kobayashi M, et al. Prediction of cerebral hyperperfusion after carotid endarterectomy using cerebral blood volume measured by perfusion-weighted MR imaging compared with single-photon emission CT. AJNR Am J Neuroradiol 2007;28:737-742.

25. Hokari M, Kuroda S, Shiga T, et al. Combination of a mean transit time measurement with an acetazolamide test increases predictive power to identify elevated oxygen extraction fraction in occlusive carotid artery diseases. J Nucl Med 2008;49:1922-1927. 


\section{FIGURE LEGENDS}

Figure 1. Regions of interest (ROIs) were placed in normal-appearing white matter (centrum semiovale or corona radiata) on the operated side. A) Fluid-attenuated inversion recuperation (FLAIR) axial imaging shows no abnormalities. B) ROIs indicated by circles are placed in normal-appearing white matter on the same slice from FLAIR imaging.

Figure 2. Preoperative mean apparent diffusion coefficient (ADC) on normal-appearing white matter (NAWM) between patients with and without postoperative transient neurological deficits. Box-and-whisker plots indicate median value (line in box) and the 25th (lower line of box) and 75th (upper line of box) percentiles. T bars indicate the 10th and 90th percentiles. P-values for comparisons between groups were calculated using the Mann-Whitney U test. Mean ADCs in patients with and without postoperative transient neurological deficits were $748.3 \pm 12 \mathrm{~mm}^{2} / \mathrm{s} \times 10^{-6}$ and $679.7 \pm 21.9 \mathrm{~mm}^{2} / \mathrm{s} \times 10^{-6}$, respectively $(P=0.0091)$. 
Table 1. Clinical characteristics of the study population

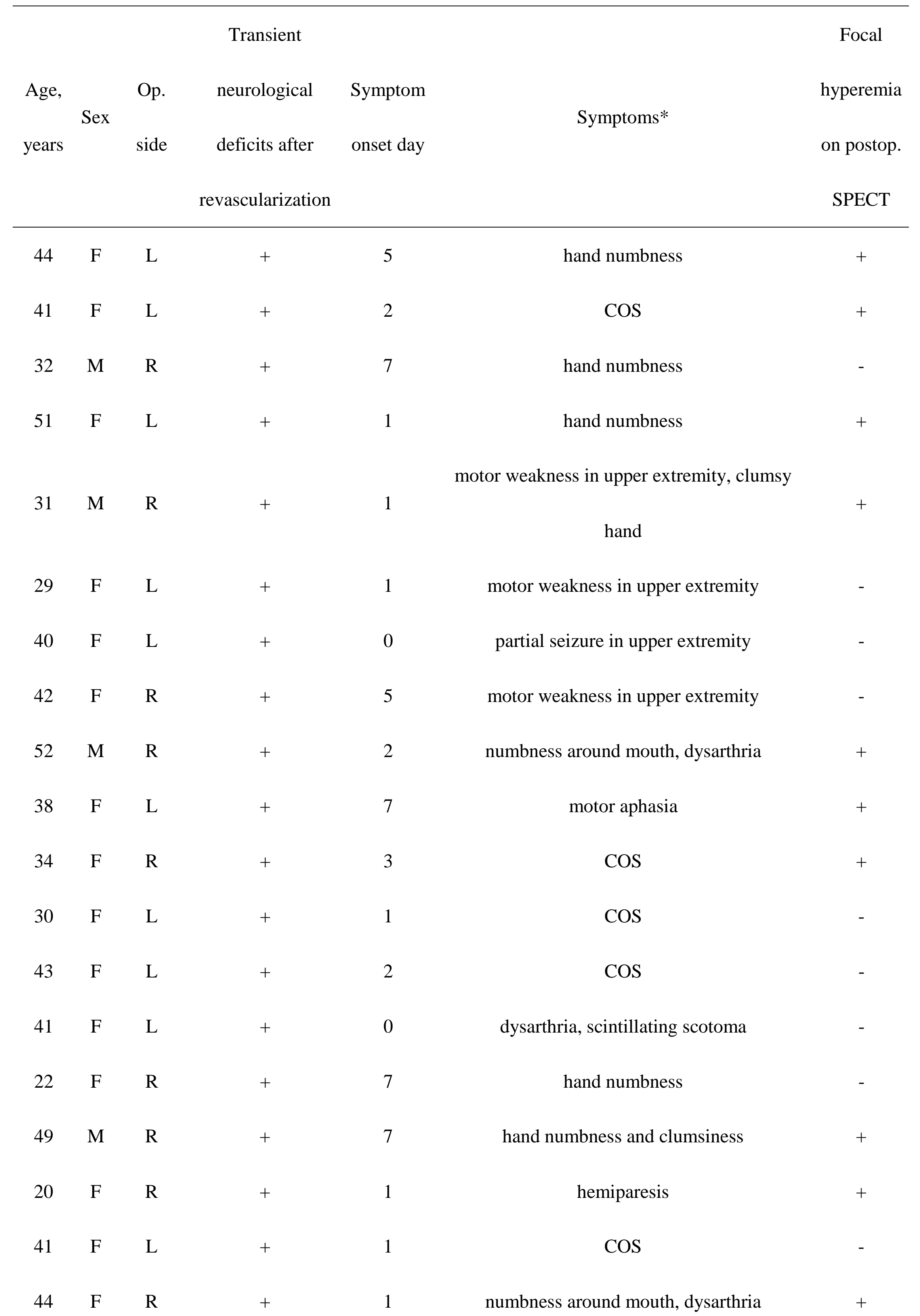




$\begin{array}{lll}43 & \mathrm{~F} & \mathrm{R} \\ 52 & \mathrm{M} & \mathrm{L} \\ 44 & \mathrm{~F} & \mathrm{~L} \\ 42 & \mathrm{M} & \mathrm{R} \\ & & \\ 38 & \mathrm{M} & \mathrm{R} \\ 28 & \mathrm{~F} & \mathrm{R} \\ & & \\ 24 & \mathrm{~F} & \mathrm{~L}\end{array}$

Op., operated; postop., postoperative; M, male; F, female; L, left; R, right; COS, cheiro-oral syndrome

* All symptoms appeared contralateral to the operated side 
Table 2. Transient neurological deficits after revascularization and mean ADC in NAWM

\begin{tabular}{|c|c|c|c|}
\hline & \multicolumn{3}{|c|}{ Transient } \\
\hline & \multicolumn{3}{|c|}{ neurological deficits } \\
\hline & \multicolumn{3}{|c|}{ after } \\
\hline & \multicolumn{2}{|c|}{ revascularization } & \multirow[b]{2}{*}{ Total } \\
\hline & Yes & No & \\
\hline \multicolumn{4}{|l|}{ Mean ADC } \\
\hline$<705.5$ & 6 & 5 & 11 \\
\hline$\geq 705.5^{*}$ & 14 & 1 & 15 \\
\hline Total & 20 & 6 & 26 \\
\hline
\end{tabular}

NAWM, normal-appearing white matter;

ADC, apparent diffusion coefficient.

$* P=0.032$ 


\section{Figure 1}
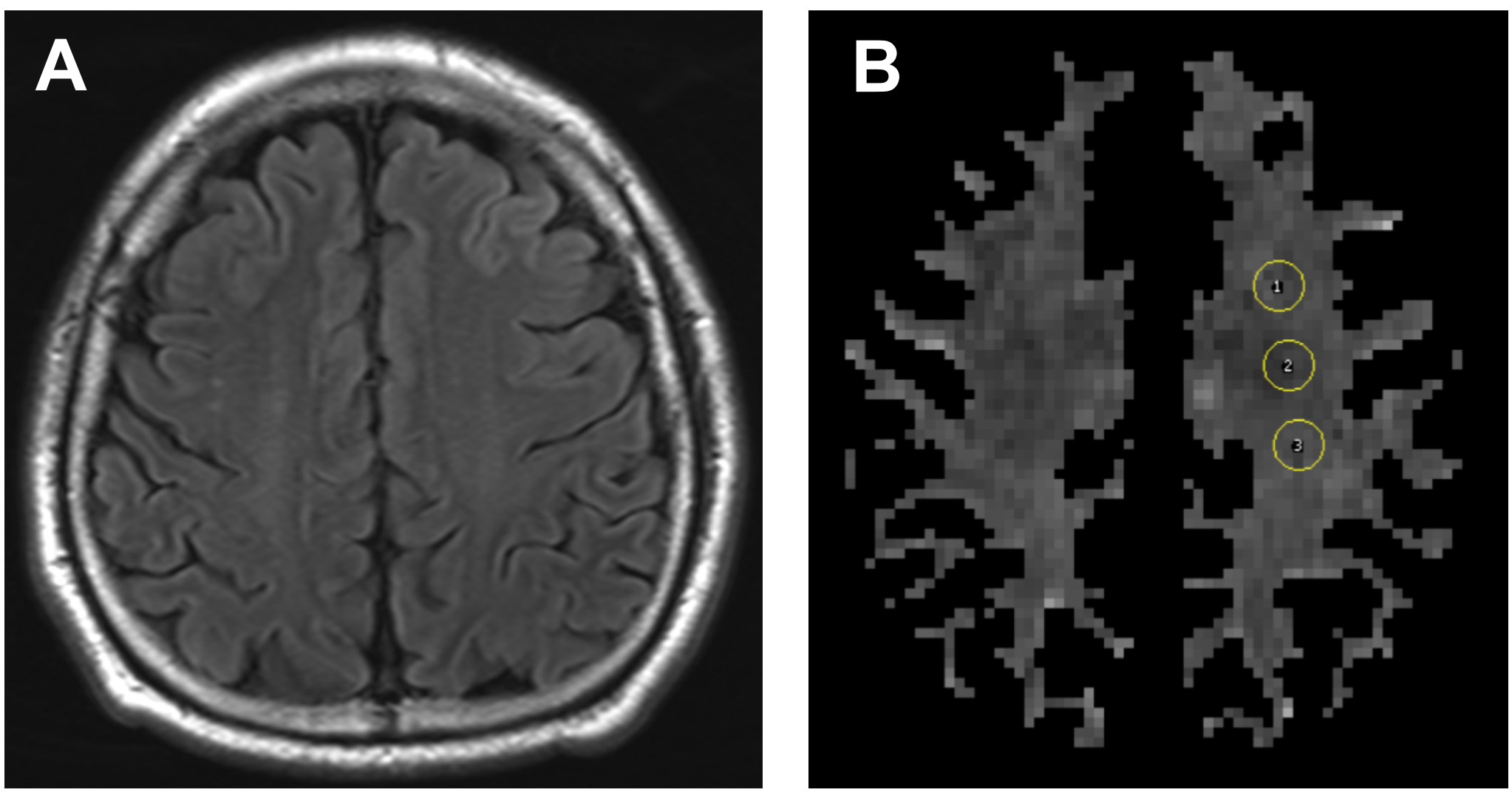
Figure 2

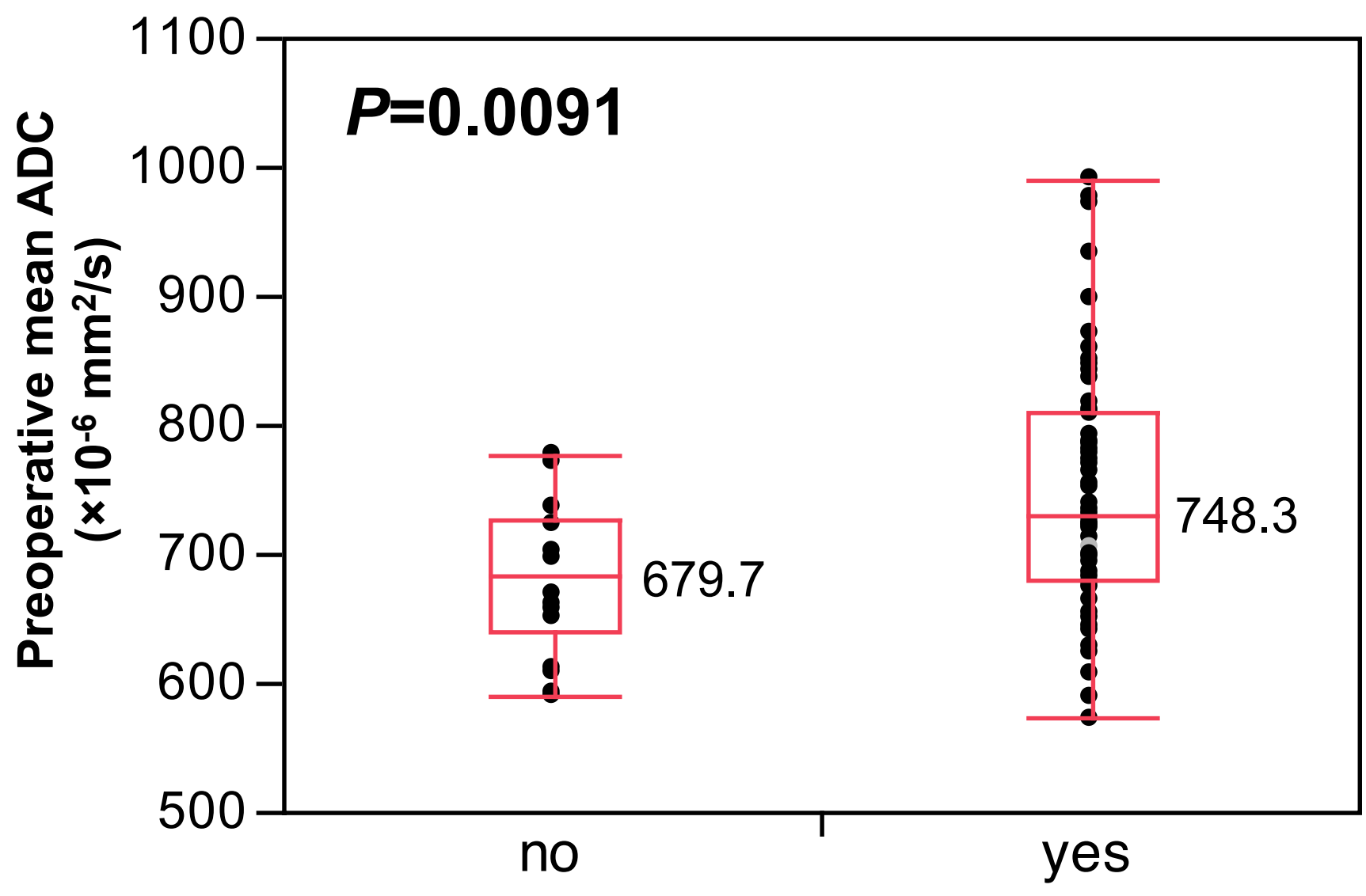

Postoperative transient neurological deficits 